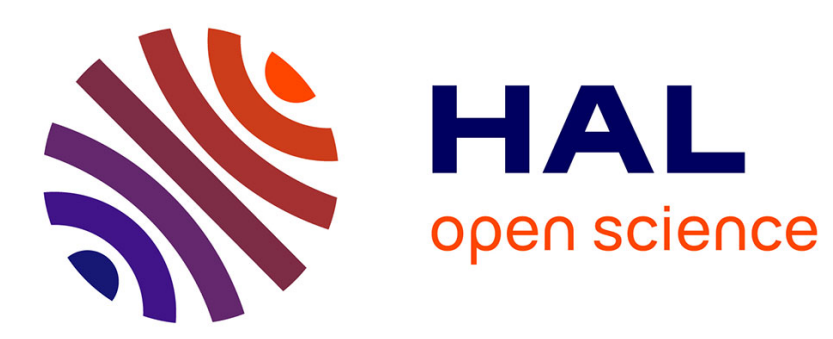

\title{
Détermination des densités de charge d'espace dans les isolants solides par la méthode de l'onde thermique
}

\author{
A. Toureille, J.-P. Reboul, P. Merle
}

\section{To cite this version:}

A. Toureille, J.-P. Reboul, P. Merle. Détermination des densités de charge d'espace dans les isolants solides par la méthode de l'onde thermique. Journal de Physique III, 1991, 1 (1), pp.111-123. 10.1051/jp3:1991254 . jpa-00248561

HAL Id: jpa-00248561

https://hal.science/jpa-00248561

Submitted on 1 Jan 1991

HAL is a multi-disciplinary open access archive for the deposit and dissemination of scientific research documents, whether they are published or not. The documents may come from teaching and research institutions in France or abroad, or from public or private research centers.
L'archive ouverte pluridisciplinaire HAL, est destinée au dépôt et à la diffusion de documents scientifiques de niveau recherche, publiés ou non, émanant des établissements d'enseignement et de recherche français ou étrangers, des laboratoires publics ou privés. 
Classification

Physics Abstracts

$61.40 \mathrm{~K}-77.50-72.20 \mathrm{~J}-66.70$

\title{
Détermination des densités de charge d'espace dans les isolants solides par la méthode de l'onde thermique
}

\author{
A. Toureille, J.-P. Reboul et P. Merle \\ Laboratoire d'Electrotechnique de Montpellier, Université des Sciences et Techniques du \\ Languedoc, place Eugène Bataillon, 34095 Montpellier Cedex 5, France
}

(Reçu le 2 février 1990, révisé le 19 juin 1990, accepté le 11 octobre 1990)

\begin{abstract}
Résumé. - Une nouvelle méthode non destructive, pour la mesure des densités de charges d'espace dans les isolants solides, est décrite. Cette méthode, dite de "l'onde thermique » est basée sur la diffusion d'un front de chaleur appliqué à une des faces de l'éprouvette et sur la dilatation non uniforme qui en résulte. A partir de la résolution de l'équation de la chaleur, nous avóns établi les relations entre le courant mesuré et les densités de charges. Nous indiquons ensuite un procédé de déconvolution permettant de calculer ces densités de charge. Quelques résultats obtenus par cette méthode, sur des plaques de polyéthylène réticulé et polypropylène sont donnés.

Abstract. - A non-destructive method for the measurement of space charge densities in solid insulating materials is described. This method called " the thermal step technique " is concerned with the diffusion of a step of heat applied to one side of the sample and with the resulting nonuniform thermal expansion. From the solution of the equation of heat, we have set up the relations between the measured current and the space charge densities. The deconvolution procedure leading to these charge densities is presented. Some results obtained with this method on XLPE and polypropylene slabs are given.
\end{abstract}

\section{Introduction.}

Dans les isolants solides soumis à des contraintes électriques élevées, un certain nombre de mécanismes encore mal connus conduisent très souvent à l'établissement d'une charge d'espace [1] qui rompt la neutralité électrique de certaines régions de l'isolant. Cette situation se maintient ou évolue lentement, même après la coupure de la contrainte électrique extérieure qui lui a donné naissance. La charge d'espace accumulée dans ces régions a pour effet d'introduire une répartition non uniforme du champ électrique qui accentue les phénomènes de dégradation et de vieillissement du matériau et réduit la durée de vie des dispositifs travaillant sous tension élevée comme les câbles et les condensateurs.

Depuis quelques années, de nombreuses études ont donc été entreprises pour élucider les mécanismes qui régissent l'établissement de cette charge d'espace afin de tenter d'en inhiber les processus ou d'agir sur leur cinétique. Mais pour aborder ces recherches, il est indispensable de pouvoir mesurer et localiser la charge d'espace stockée dans un isolant, de 
façon à mettre en évidence l'influence éventuelle de chaque facteur physique ou l'aptitude propre de chaque matériau. Diverses méthodes de mesure ont déjà été proposées. Nous rappelons brièvement, dans la deuxième partie de ce travail, les principales d'entre elles.

Nous avons, quant à nous, développé et perfectionné une nouvelle méthode de mesure non destructive, dite de «l'onde thermique », dont nous avons déjà présenté les premiers résultats [2-3] et qui s'applique aussi bien à des structures planes telles que des feuilles ou des plaques d'isolant, qu'à des structures cylindriques comme les câbles [4]. La description complète de cette méthode et le développement de la théorie et des calculs qu'elle nécessite, dans le cas de structures planes, font l'objet de la troisième partie de ce travail.

Enfin dans la quatrième partie, nous donnóns quelques distributions de charge d'espace mesurées par cette méthode sur des plaques de polyéthylène réticulé et de polypropylène.

\section{Principales méthodes de mesure des charges d'espace.}

Les techniques de courants stimulés thermiquement [5] ont connu un vif succès et ont été longtemps les seules à apporter quelques renseignements précieux sur les mécanismes de piégeage, de dépiégeage, de recombinaison et de transport des charges. Malheureusement, elles permettent difficilement la détermination quantitative des charges et encore plus difficilement leur localisation, même en améliorant leur précision [6].

Les premières tentatives concernant la localisation des charges d'espace sont dues à Collins [7]. Sa méthode consistait à appliquer une impulsion de lumière sur une face métallisée de l'électret, ce qui prodứisait une courte impulsion de chaleur., La. propagation de cette impulsion de chaleur déplaçait la charge d'espace et entraînait dés väriations de potentiel entre les faces de l'électret. Le calcul des densités de charge d'espace à partir des variations de potentiel est ardu et conduit à de grandes imprécisions [8].

D'autres méthodes, basées sur un principe analogue et utilisant la propagation d'une onde de compression, ont été développées par la suite. Plusieurs variantes ont été proposées. Elles se distinguent surtout par la façon de produire l'onde de compression. Initialement, on a utilisé un tube à onde de choc [9], puis plus tard, des impulsions laser [10-11-12]. On s'est servi également des impulsions laser pour produire des échauffements de courte dúrée [13] ou des échauffements modulés [14].

Les limites des méthodes développées actuellement concernent :

- Le pouvoir de résolution, car la durée des impulsions doit rester faible devant le temps de transit de la perturbation à travers l'épaisseur de l'échantillon.

- La déformation de l'impulsion dont il faudrait tenir compte, surtout pour des échantillons épais afin d'éviter l'imprécision des résultats.

- Les difficultés d'étalonnage qui font que les densités de charge d'espace sont souvent données en unités arbitraires.

- Le-caractère destructif de certaines techniques qui engendrent des échauffements et provoquent la disparition des charges d'espace suivant un processus de thermostimulation.

\section{La méthode de l'onde thermique.}

3.1 PrinCIPE. - Considérons donc une plaque d'isolant d'épaisseur constante $D$, ' placée entre deux électrodes planes et parallèles, de surface $S$, reliées entre elles par un court-circuit ou par un appareil de mesure du courant. L'épaisseur $D$ étant petite devant les autres dimensions, l'étude s'effectuera suivant la direction $x$ perpendiculaire aux électrodes (modèle unidimensionnel).

Soit une charge $Q i$ située à l'intérieur du matériau, dans une couche plane infiniment mince, de surface $S$, parallèle aux électrodes et à la distance $x$ et $D-x$ de celles-ci. Par 
influence totale, cette charge $Q i$ induit, sur les électrodes, des charges images $Q i_{1}$ et $Q i_{2}$ qui dépendent, entre autres, des distances $x$ et $D-x$.

La pénétration d'une onde de chaleur à partir de l'une des électrodes et la dilatation qui l'accompagne fait varier les valeurs relatives de ces distances et modifie l'équilibre des charges images $Q i_{1}$ et $Q i_{2}$. Comme par ailleurs la somme $Q i_{1}+Q i_{2}$ doit rester constante. Les échanges de charges entre les électrodes se traduisent par un courant dans le circuit extérieur que l'on peut mesurer et enregistrer.

Si l'on connaît, à chaque instant, la valeur du courant et la répartition de la température dans l'échantillon, on peut retrouver la valeur de la charge $Q i$ et sa position $x$, comme nous allons le voir plus. loin.

3.2 TECHNIQUE D'OBTENTION D'UNE ONDE THERMIQUE CONTROLÉE. - La propagation d'une onde de chaleur accompagnée d'une dilatation inhomogène peut être obtenue de la façon suivante :

La cellule de mesure étant initialement à la température $T_{0}$, à l'instant $t=0$, on fait circuler un liquide à la température $T_{1}>T_{0}$ dans un radiateur situé contre une des électrodes (source chaude). L'autre électrode par sa masse et son inertie thermique est supposée constituer la source froide.

L'amplitude du courant dans le circuit extérieur et par suite la précision de la méthode sont directement proportionnelles à la différence de température $\Delta T_{0}=T_{1}-T_{0}$, comme nous le verrons dans la théorie développée ci-après. Mais en fait, l'intervalle $\Delta T_{0}$ doit se situer dans une zone où les courants thermostimulés et les courants de conduction sont négligeables ce qui se traduira par la reproductibilité de 2 mesures faites successivement.

Pour notre étude sur le polyéthylène, nous avons choisi de placer la cellule de mesure dans une étuve réfrigérée à $T_{0}=-10^{\circ} \mathrm{C}$ et de faire circuler dans le radiateur d'électrode un liquide provenant d'un réservoir à température ambiante $\left(T_{1}=+20{ }^{\circ} \mathrm{C}\right)$. Nous obtenons ainsi très commodément une différence de température de $30^{\circ} \mathrm{C}$ dans une plage où le polyéthylène ne fournit pas de courants thermostimulés, comme nous avons eu l'occasion de le vérifier [6-15], et ne présente pas, par conséquent, de changements de phase.

Puisque cette opération ne décharge pas l'échantillon, la mesure'n'est pas destructive. Elle peut donc être recommencée à n'importe quel moment, sitôt que l'ensemble a été à nouveau refroidi à $-10{ }^{\circ} \mathrm{C}$.

Sur le polypropylène, cette plage semble également convenir puisque les résultats de 2 mesures successives sont reproductibles.

\subsection{ETABLISSEMENT DE L'EXPRESSION THÉORIQUE DU COURANT DANS LE CIRCUIT EXTÉ- RIEUR.}

3.3.1 Hypothèses et notations. - L'ensemble de la cellule est supposé à la température uniforme $T_{0}$.

A l'instant $t=0$, une source chaude de température $T_{1}$ apparaît à l'abscisse 0 (origine des abscisses).

La première interface électrode-échantillon est à l'abscisse $x_{0} ; x_{0}$ représente une épaisseur équivalente de matériau entre la source chaude et la première face de l'échantillon.

La deuxième électrode est à l'abscisse $x_{0}+D$.

La source froide est à l'abscisse $L ; L-x_{0}-D$ représente une épaisseur équivalente de matériau entre la deuxième face de l'échantillon et la source froide.

L'équation de la chaleur est : $\partial^{2} T / \partial x^{2}=W \partial T / \partial t$, avec $W=\mu C / \lambda$, $\mu$ étant la masse volumique de l'isolant, $C$ sa chaleur massique et $\lambda$ sa conductibilité thermique. Dans le domaine de variation de la température choisi et pour les matériaux que'nous utilisons $W$ évolue très peu (quelques \%) ce que nous avons négligé. 
On remarquera qu'une épaisseur $x$ de matériau ayant un paramètre thermique $W$ peut être remplacé par une épaisseur $x^{\prime}$ de matériau ayant un paramètre thermique $W^{\prime}$, sans que la solution de l'équation de la chaleur soit modifiée, pourvu que $W / W^{\prime}=\left(x^{\prime} / x\right)^{2}$. Il n'y a donc aucun inconvénient à raisonner sur des épaisseurs équivalentes constituées du même matériau isolant que l'éprouvette, situées de part et d'autre de celle-ci, de façon à « homogénéiser » l'intervalle entre la source chaude et la source froide.

3.3.2 Solution de l'équation de la chaleur dans le cas d'un échelon de température d'amplitude $\Delta T_{0}=T_{1}-T_{0}$. - Si à l'instant $t=0$, une source chaude de température $T_{1}$ apparaît à l'abscisse 0 , alors qu'une source froide maintient une température $T_{0}$ à l'abscisse $L$, la résolution de l'équation de la chaleur donne l'expression de la température en un point d'abscisse $x$ comprise entre 0 et $L$, soit :

$$
T(x, t)=T_{0}+\Delta T_{0}(1-x / L)-2\left(\Delta T_{0} / \pi\right) \sum_{1}^{\infty}(1 / n) \exp \left(-t / \tau_{n}\right) \sin (n \pi x / L)
$$

avec

$$
\tau_{n}=W L^{2} / n^{2} \pi^{2}
$$

3.3.3 Expression des conditions électriques imposées au système. - Pour une charge $Q i$ située dans un plan parallèle aux électrodes, à l'abscisse $x$, la condition d'influence totale impose que la somme des charges soit nulle :

$$
Q i_{1}+Q i_{2}+Q i=0
$$

La condition «électrodes en court-circuit » impose quie la circulation du champ électrique $E$ sur l'intervalle $\left(x_{0}, x_{0}+D\right)$ soit nulle également. Par ailleurs, l'induction électrique $\varepsilon E$ doit être constante en l'absence de charges, de sorte que l'on peut écrire :

- sur l'intervalle $\left(x_{0}, x\right), \quad \varepsilon E=Q i_{1}$

- sur l'intervalle $\left(x, x_{0}+D\right), \quad \varepsilon E=Q i_{1}+Q i=-Q i_{2}$.

Il s"ensuit que :

$$
\int_{x_{0}}^{x}(1 / \varepsilon) Q i_{1} \cdot \mathrm{d} u+\int_{x}^{x_{0}+D}(1 / \varepsilon)\left(Q i_{1}+Q i\right) \mathrm{d} u=0
$$

D'où l'on tire :

$$
\begin{aligned}
& Q i_{1}=-Q i \int_{x}^{x_{0}+D}(1 / \varepsilon) \mathrm{d} u / \int_{x}^{x_{0}+D}(1 / \varepsilon) \mathrm{d} u \\
& Q i_{2}=-Q i \int_{x_{0}}^{x}(1 / \varepsilon) \mathrm{d} u / \int_{x_{0}}^{x_{0}+D}(1 / \varepsilon) \mathrm{d} u
\end{aligned}
$$

3.3.4 Prise en compte de l'influence de la température. - A cause de la dilatation, les distances ou les épaisseurs dépendent de la température. On écrira donc:

$$
\mathrm{d} u=\mathrm{d} u_{0}\left(1+\alpha_{\mathrm{D}}\left(T-T_{0}\right)\right)
$$

$\alpha_{\mathrm{D}}$ étant le coefficient de dilatation linéaire du matériau dans la direction $x$ et dans les conditions de l'expérience. 
La permittivité $\varepsilon$ dépend, elle aussi, de la température, ce que l'on traduira par:

$$
\varepsilon=\varepsilon_{\mathrm{p}}\left(1+\alpha_{\mathrm{p}}\left(T-T_{0}\right)\right),
$$

$\alpha_{\mathrm{p}}$ étant le coefficient de variation de $\varepsilon$ avec la température, dans les conditions de l'expérience.

La quantité $\mathrm{d} u / \varepsilon$ qui intervient dans les expressions (3) et (4) s'écrit, au premier ordre, en posant $\alpha_{\mathrm{D}}-\alpha_{\mathrm{p}}=\alpha$ et $T-T_{0}=\vartheta$ :

$$
\mathrm{d} u / \varepsilon \simeq \mathrm{d} u_{0}(1+\alpha \vartheta) / \varepsilon_{\mathrm{p}} .
$$

L'expression (4) devient alors :

$$
Q i_{2} \simeq-Q i(1 / D)\left(x-x_{0}\right)\left(1+\left(\alpha /\left(x-x_{0}\right)\right) \int_{x_{0}}^{x} \vartheta \mathrm{d} u_{0}-(\alpha / D) \int_{x_{0}}^{x_{0}+D} \vartheta \cdot \mathrm{d} u_{0}\right) .
$$

3.3.5 Expression du courant. - L'expression du courant qui apparaîtra dans le circuit extérieur, dans l'hypothèse de la seule charge d'espace $Q i$, sera :

$$
i(t)=-\partial Q i_{2} / \partial t
$$

Soit :

$$
i(t)=Q i(1 / D) \alpha\left(\int_{x_{0}}^{x}(\partial \vartheta / \partial t) \mathrm{d} u_{0}-\left(\left(x-x_{0}\right) / D\right) \int_{x_{0}}^{x_{0}+D}(\partial \vartheta / \partial t) \mathrm{d} u_{0}\right)
$$

Dans le cas d'une charge d'espace de densité équivalente $\rho(x)$ (tenant compte des charges libres et liées), répartie dans l'épaisseur du matériau, on remplacera $Q i$ par $S \int_{x_{0}}^{x_{0}+D} \rho(x) \cdot \mathrm{d} x$. D'où l'expression du courant total :

$$
I(t)=S \alpha(1 / D) \int_{x_{0}}^{x_{0}+D} \rho(x)\left(\int_{x_{0}}^{x}(\partial \vartheta / \partial t) \mathrm{d} u_{0}-\left(\left(x-x_{0}\right) / D\right) \int_{x_{0}}^{x_{0}+D}(\partial \vartheta / \partial t) \mathrm{d} u_{0}\right) \mathrm{d} x
$$

Ce courant est de la forme :

$$
I(t)=S \alpha(1 / D) \int_{x_{0}}^{x_{0}+D} \rho(x) \cdot f(x, t) \cdot \mathrm{d} x,
$$

avec $\quad f(x, t)=\int_{x_{0}}^{x}(\partial \vartheta / \partial t) \cdot \mathrm{d} u_{0}-\left(\left(x-x_{0}\right) / D\right) \int_{x_{0}}^{x_{0}+D}(\partial \vartheta / \partial t) \cdot \mathrm{d} u_{0}$

Il s'agit d'une équation intégrale où $\rho(x)$ est l'inconnue et $f(x, t)$ le noyau.

\subsection{RÉSOLUTION DE L'ÉQUATION INTÉGRALE.}

3.4.1 Changement de variable. $-\vartheta=T-T_{0}$ est une fonction de $x$ et de $t$ donnée par la relation (1). On en tire :

$$
\partial \vartheta / \partial t=2 \Delta T_{0} \pi\left(1 / W L^{2}\right) \sum_{1}^{\infty} n \exp \left(-t / \tau_{n}\right) \sin (n \pi x / L)
$$


L'expression de $f(x, t)$ devient alors:

$$
f(x, t)=2 \Delta T_{0}(1 / W L) \sum_{1}^{\infty} \exp \left(-t / \tau_{n}\right) \cdot g(x, n),
$$

avec

$$
\begin{aligned}
g(x, n)=\cos \left(n \pi x_{0} / L\right)-\cos (n \pi x / L)-\left(x-x_{0}\right)(1 / D) \times & . \\
& \times\left(\cos \left(n \pi x_{0} / \dot{L}\right)-\cos \left(n \pi\left(x_{0}+D\right) / L\right)\right) .
\end{aligned}
$$

Notons que pour $x=x_{0}$ et $x=x_{0}+D$, la fonction $g(x, \bar{n})$ est nulle et par suite la fonction $f(x, t)$ est nulle aussi. Cette remarque permet de simplifier le résultat d'une intégration par parties de la quantité $\int_{x_{0}}^{x_{0}+D} \rho(x) . f(x, t) . \mathrm{d} x$ où $\rho(x)=\partial(\varepsilon E) / \partial x, \varepsilon E$ étant l'induction électrique.

On obtient alors :

$$
I(t)=-S \alpha(1 / D) \int_{x_{0}}^{x_{0}+D} \varepsilon E \cdot(\partial f / \partial x) \cdot \mathrm{d} x
$$

Mais $\quad \partial f / \partial x=2 \Delta T_{0}(1 / W L) \sum_{1}^{\infty} \exp \left(-t / \tau_{n}\right) \cdot \partial g / \partial x$, et par ailleurs: $\partial g / \partial x=$ $(n \pi / L) \sin (n \pi x / L)+K, K$ quantité indépendante de $x$. Donc :

$$
\begin{aligned}
I(t)=-S \alpha(1 / D) 2 \Delta T_{0}(1 / W L) \int_{x_{0}}^{x_{0}+D} & \varepsilon E \times \\
& \times \sum_{1}^{\infty}\left(\exp \left(-t / \tau_{n}\right) \cdot((n \pi / L) \sin (n \pi x / L)+K)\right) \mathrm{d} x .
\end{aligned}
$$

Or, au premier ordre,

$$
\int_{x_{0}}^{x_{0}+D} \varepsilon E \cdot \mathrm{d} x \simeq \varepsilon \int_{x_{0}}^{x_{0}+D} E \cdot \mathrm{d} x=0, \quad \text { (condition de court-circuit). }
$$

Il restera donc :

$$
I(t)=-\left(2 S \alpha \Delta T_{0} / W L D\right) \int_{x_{0}}^{x_{0}+D} \varepsilon E \cdot \sum_{1}^{\infty}\left(\exp \left(-t / \tau_{n}\right) \cdot(n \pi / L) \sin (n \pi x / L)\right) \mathrm{d} x \cdot(7)
$$

3.4.2 Résolution par les séries de Fourier. - Soit une fonction $h(x)$, définie sur l'intervalle $(0, L)$, égale à $\varepsilon E / \varepsilon_{\mathrm{p}}$ sur l'intervalle $\left(x_{0}, x_{0}+D\right)$ et nulle ailleurs.

Dans l'expression (7), il n'y a donc aucun inconvénient à remplacer $\varepsilon E$ par $\varepsilon_{\mathrm{p}} h(x)$ et à étendre l'intervalle d'intégration à $(0, L)$, soit":

$$
I(t)=-\left(2 S \alpha \Delta T_{0} \varepsilon_{\mathrm{p}} / W L D\right) \int_{0}^{L} h(x) \cdot \sum_{1}^{\infty}\left(\exp \left(-t / \tau_{n}\right) \cdot(n \pi / L) \sin (n \pi x / L)\right) \mathrm{d} x .
$$

On remarquera que la valeur moyenne de $h(x)$ est nulle sur l'intervalle $\left(x_{0}, x_{0}+D\right)$ en raison du court-circuit et qu'elle est nulle également sur l'intervalle élargi $(0, L)$. En outre on a $h(x)=0$ pour $x=0$ et pour $x=L$. Dans ces conditions, la décomposition en série de Fourier de la fonction $h(x)$ peut se mettre sous la forme :

$$
h(x)=E_{0} \sum_{1}^{\infty} b_{m} \sin (m \pi x / L) \text {. }
$$


En remplaçant $h(x)$ par sa série de Fourier dans l'expression de $I(t)$ et en développant, on obtient des termes du type $\sin (n \pi x / L) \cdot \sin (m \pi x / L)$. L'intégrale de ces termes sur l'intervalle $(0, L)$ est nulle sauf quand $n=m$, auquel cas, elle vaut $L / 2$.

Ceci permet d'écrire :

$$
I(t)=-\left(\pi S \alpha \Delta T_{0} E_{0} \varepsilon_{\mathrm{p}} / W L D\right) \cdot \sum_{1}^{\infty} n \cdot b_{n} \cdot \exp \left(-t / \tau_{n}\right),
$$

ou encore :

$I(t)=\sum_{1}^{\infty} I_{n}(t)$

avec

$$
I_{n}(t)=I_{0} \cdot n \cdot b_{n} \cdot \exp \left(-t / \tau_{n}\right) \quad \text { et } \quad I_{0}=-\pi S \alpha E_{0} \Delta T_{0} \varepsilon_{\mathrm{p}} / W L D
$$

\subsection{ETALONNAGE DU DISPOSITIF EXPÉRIMENTAL.}

3.5.1 Principe. - Dans la théorie ci-dessus, l'expression du courant dans le circuit extérieur fait intervenir à la fois des caractéristiques du matériau et des paramètres liés à la cellule de mesure et aux conditions expérimentales $\left(\alpha, W, x_{0}, L\right)$. Ces quantités doivent être connues avec précision. Nous avons donc mis au point une mesure d'étalonnage permettant de les déterminer.

Cette mesure s'effectue de préférence sur l'éprouvette qui doit être étudiée, avant de la charger, ou alors sur une' éprouvette identique. Dans tous les cas, il ne doit subsister aucune charge d'espace dans le matériau. On s'en assure en appliquant la méthode de l'onde thermique qui ne doit donner aucun courant. Dans le cas contraire, l'échantillon courtcircuité est chauffé dans une étuve, de façon à le décharger complètement par thermostimulation.

L'échantillon se trouvant dans la cellule de mesure et ayant été refroidi à $-10{ }^{\circ} \mathrm{C}$, on lui applique un champ continu constant et stable, de l'ordre du kv/mm. Dans de telles conditions d'expérimentation, il a été vérifié qu'il n'y a pas d'injection de charges et que l'éprouvette se comporte comme un condensateur plan ayant une tension constante à ses bornes, c'est-à-dire que toute la charge est localisée aux interfaces isolant-électrodes.

On applique l'échelon de température dans les conditions habituelles de la méthode et on enregistre le courant qui se met à circuler dans le circuit extérieur, entre l'éprouvette et la source de tension.

3.5.2 Expression du courant d'étalonnage. - Soit $C$ la capacité de l'éprouvette. Cette capacité peut être considérée comme la somme de condensateurs élémentaires d'épaisseur du, placés en série. On a donc:

$$
1 / C=\int_{x_{0}}^{x_{0}+D}(1 / \varepsilon S) \mathrm{d} u
$$

En remplaçant $\mathrm{d} u / \varepsilon$ par l'expression (5), on obtient :

$$
1 / C \simeq \int_{x_{0}}^{x_{0}+D}\left(1 / \varepsilon_{\mathrm{p}} S\right)(1+\alpha \vartheta) \mathrm{d} u_{0}
$$

ce qui donne :

$$
C \simeq S \varepsilon_{\mathrm{p}} / D-\left(S \varepsilon_{\mathrm{p}} / D^{2}\right) \int_{x_{0}}^{x_{0}+D} \alpha \vartheta \mathrm{d} u_{0}
$$


La charge de cette capacité, sous la tension constante $V$, vaut $C V$; d'où l'expression du courant mesuré dans le circuit extérieur :

$$
I_{\mathrm{e}}(t)=V \cdot \partial C / \partial t=-\left(S \varepsilon_{\mathrm{p}} \alpha V / D^{2}\right) \int_{x_{0}}^{x_{0}+D}(\partial \vartheta / \partial t) \cdot \mathrm{d} u_{0}
$$

En remplaçant $\partial \vartheta / \partial t$ par son expression tirée de (6), on obtient :

$$
\begin{aligned}
I_{\mathrm{e}}(t)=-2 \Delta T_{0} S \varepsilon_{\mathrm{p}} \alpha V\left(1 / W L D^{2}\right) & \times \\
& \times \sum_{1}^{\infty} \exp \left(-t / \tau_{n}\right)\left(\cos \left(n \pi x_{0} / L\right)-\cos \left(n \pi\left(x_{0}+D\right) / L\right)\right) .
\end{aligned}
$$

Posons

$$
I_{\mathrm{e} 0}=-2 \Delta T_{0} S \varepsilon_{\mathrm{p}} \alpha V / W L D^{2} \text { et } c_{n}=\cos \left(n \pi x_{0} / L\right)-\cos \left(n \pi\left(x_{0}+D\right) / L\right) .
$$

On a alors :

$$
I_{\mathrm{e}}(t)=I_{\mathrm{e} 0} \sum_{1}^{\infty} c_{n} \exp \left(-t / \tau_{n}\right)
$$

3.6 MÉTHOdE DE TRAITEMENT NUMÉRIQUE ET VÉRIFICATION DES RÉSULTATS. - Le courant dans le circuit extérieur est mesuré par un électromètre Keithley $610 \mathrm{C}$ dont la sortie analogique fournit une tension maximum de 3 volts pour le maximum de déviation de l'appareil. Une carte analogique-numérique, à plusieurs entrées, permet d'échantillonner le signal de sortie de l'électromètre ainsi que les signaux fournis par des détecteurs de température signalant l'arrivée du liquide dans le radiateur (repérage de l'instant $t=0$ ). Ces signaux sont enregistrés sur un ordinateur, puis ils sont repris et traités en tenant compte de la théorie développée ci-dessus.

Nous avons vu que les courants obtenus, aussi bien dans la mesure que dans l'étalonnage, sont des sommes de termes exponentiels où interviennent des constantes de temps qui dépendent toutes de $\tau_{1}$ qui est la plus grande : $\tau_{1}=4 \tau_{2}=9 \tau_{3}, .$. , etc., d'après la relation (2).

La détermination de ces constantes de temps ne présente pas de difficultés puisque $\tau_{1}$ peut être calculée avec beaucoup de précision par l'étude de la décroissance du courant lorsque $t$ est grand. Pour le courant d'étalonnage, on a ainsi :

$$
I_{\mathrm{e}}(t) \simeq I_{\mathrm{e} 0} c_{1} \exp \left(-t / \tau_{1}\right) .
$$

Pour nos échantillons dont l'épaisseur est de $2 \mathrm{~mm}$, nous avons trouvé des valeurs de $\tau_{1}$ de l'ordre de 10 secondes.

Avec une valeur aussi grande de $\tau_{1}$, le nombre des coefficients $c_{n}$ qui peuvent être déterminés est important. En effet, le temps de réponse de notre détecteur étant de 20 millisecondes, les termes dont la constante de temps $\tau_{n}=\tau_{1} / n^{2}$ est supérieure à 20 millisecondes sont pris en compte par l'appareillage et leur contribution apparaît dans la courbe expérimentale du courant. Ceci correspond environ à une vingtaine de termes, à condition toutefois que leur amplitude soit supérieure au bruit de fond des appareils qui est de l'ordre de 0,05 picoampère. On arrive à déterminer une quinzaine de coefficients $c_{n}$ de la série de Fourier dans l'expression du courant (8), en utilisant un algorithme répétitif convergent qui permet de retrouver la courbe expérimentale avec précision en faisant une somme de termes exponentiels de constantes de temps connues. Les valeurs des 
$c_{n}$ et de $\tau_{1}$ servent à déterminer $x_{0}, L, W$ et $\alpha$. On notera que nous avons beaucoup plus d'équations que d'inconnues, ce qui permet de vérifier l'exactitude de la théorie.

Pour traiter le courant expérimental donné par un échantillon renfermant de la charge d'espace, la méthode est analogue. On reconstitue le signal par une somme de termes exponentiels dont les constantes de temps sont connues, en commençant par la fin de la courbe, où intervient seulement le terme en $\tau_{1}$, puis en remontant le temps et en rajoutant les contributions successives relatives aux termes en $\tau_{2}, \tau_{3}, \ldots, \tau_{n}$, jusqu'à la limite significative imposée par les appareils de mesure. On obtient ainsi, suivant l'amplitude du signal mesuré, et l'épaisseur de l'échantillon entre 12 et 18 coefficients $b_{n}$.

La fonction $h(x)$ est donc reconstituée par les 12 ou 18 premiers termes de sa décomposition en série, ce qui donne une précision suffisante, sauf aux endroits où la fonction présente des discontinuités ou des variations brutales, ce qui est le cas sur les électrodes.

Les valeurs de $\rho(x)$ sont déduites par dérivation de $h(x)$.

A partir de la densité $\rho(x)$ qui est ainsi déterminée, une simulation que nous avons également mise au point, reconstitue le courant théorique $I_{\mathrm{s}}(t)$ ' que cette densité $\rho(x)$ donnerait,' si on lui appliquait l'échelon de température $\vartheta$, dans les conditions de l'expérience. La concordance entre le courant simulé et le courant expérimental est la meilleure preuve de la précision des résultats et de l'efficacité de la méthode de déconvolution.

Afin de vérifier la validité de la méthode, l'onde thermique a été appliquée tour à tour des 2 côtés de l'échantillon : ainsi les distributions trouvées ont pu être contrôlées.

\section{Résultats sur des plaques.}

Nous avons appliqué la méthode de londe thermique que nous venons de décrire, à des plaques de polyéthylène réticulé chimiquement ( $P R C)$ et de polypropylène ( $P P$ ) que nous avions préalablement «polarisées ».

Les dimensions, les conditions de polarisation et de conservation des éprouvettes sont les suivantes :

- Plaques carrées de $20 \mathrm{~cm}$ de côté.

- Epaisseur de l'isolant (entre électrodes) $D: 1,5$ à $4 \mathrm{~mm}$.

- Nature des électrodes : disques de polymère semiconducteur de $0,20 \mathrm{~mm}$ d'épaisseur et $40 \mathrm{~mm}$ de diamètre, appliqués à chaud au centre de la plaque.

- Champ de polarisation $E_{\mathrm{p}}: 10 \mathrm{kV} / \mathrm{mm}$.

- Temps d'application $t_{\mathrm{a}}: 3$ jours.

- Température de polarisation $T_{\mathrm{p}}: 70^{\circ} \mathrm{C}$.

- Conservation des éprouvettes en court-circuit et à température ambiante après polarisation, pendant un temps $t_{c c}$, avant de faire la mesure.

Les figures 1 et 2 donnent les répartitions respectives de la densité de charge d'espace et du champ électrique qui subsistent dans l'épaisseur d'une plaque de PRC $(1,80 \mathrm{~mm})$, la cathode étant à $x=0$ avec $t_{\mathrm{cc}}=3$ jours.

On remarque la présence d'homocharges près de la cathode, suivie d'une couche de signe contraire, alors qu'à l'anode peu de charges apparaissent.

Les figures 3 et 4 donnent les répartitions respectives de la densité de charge d'espace et du champ électrique qui subsistent dans l'épaisseur d'une plaque de PP $(4,0 \mathrm{~mm})$, la cathode étant à $x=0$ avec $t_{\mathrm{cc}}=2$ heures.

Bien sûr, en fonction de $t_{\mathrm{cc}}$ croissant les charges disparaissent par recombinaison comme nous avons pu le vérifier; mais ce phénomène est si lent qu'il ne donne pas de courant mesurable à température ambiante. 


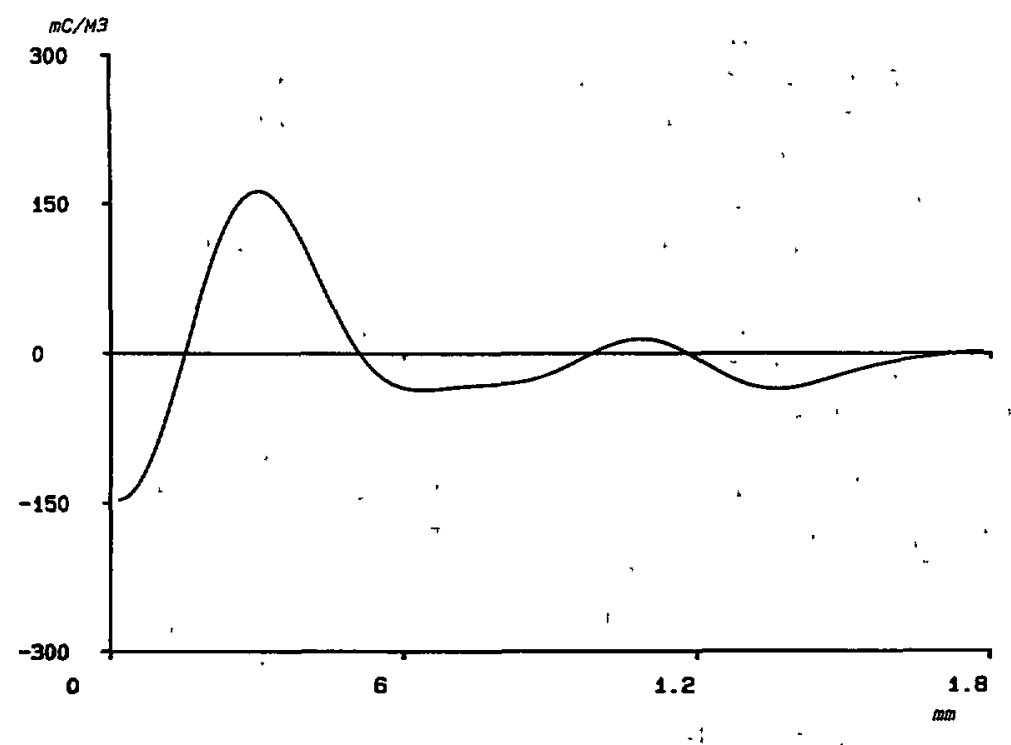

Fig. 1. - Distribution de charge d'espace dans un échantillon de PRC (polyéthylène réticulé) épais de $1,80 \mathrm{~mm}$ conservé 3 jours en court-circuit ; conditions de formation : voltage appliqué $18 \mathrm{kV}$ pendant 3 jours à $70^{\circ} \mathrm{C}$. Cathode à $x=0$ et anode à $x=1,80 \mathrm{~mm}$. Stockage à l'ambiante.

[Distribution of space charge density obtained in a $1.8 \mathrm{~mm}$ thick PRC sample for a short circuiting storage time $t_{\mathrm{cc}}=3$ days. Forming conditions: Applied voltage $18 \mathrm{kV}$ during 3 days at $70^{\circ} \mathrm{C}$. Cathode at $x=0$, Anode at $x=1.8 \mathrm{~mm}$. Storage at room temperature.]

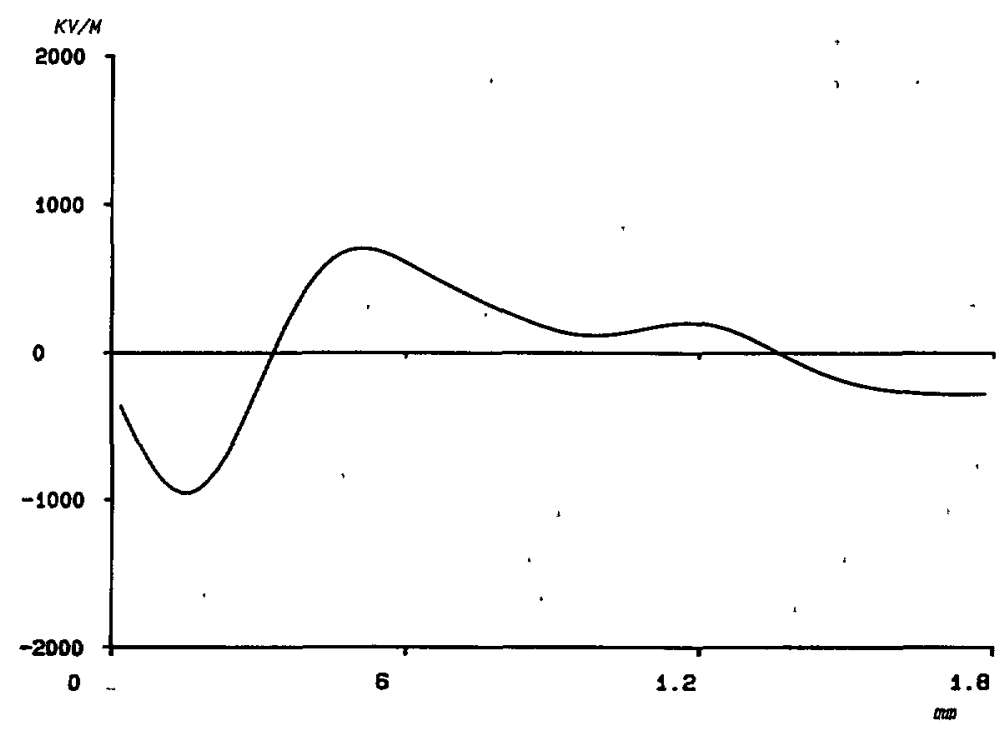

Fig. 2. - Distribution du champ électrique dans un échantillon de PRC épais de 1,80 mm conservé 3 jours en court-circuit; conditions de formation : voltage appliqué $18 \mathrm{kV}$ pendant 3 jours à $70^{\circ} \mathrm{C}$. Cathode à $x=0$ et anode à $x=1,80 \mathrm{~mm}$. Stockage à l'ambiante.

[Distribution of electric field obtained in a $1.8 \mathrm{~mm}$ thick PRC sample for a short circuiting storage time $t_{\mathrm{cc}}=3$ days. Forming conditions: Applied voltage $18 \mathrm{kV}$ during 3 days. at $70{ }^{\circ} \mathrm{C}$. Cathode at $x=0$, Anode at $x=1.8 \mathrm{~mm}$. Storage at room temperature.] 


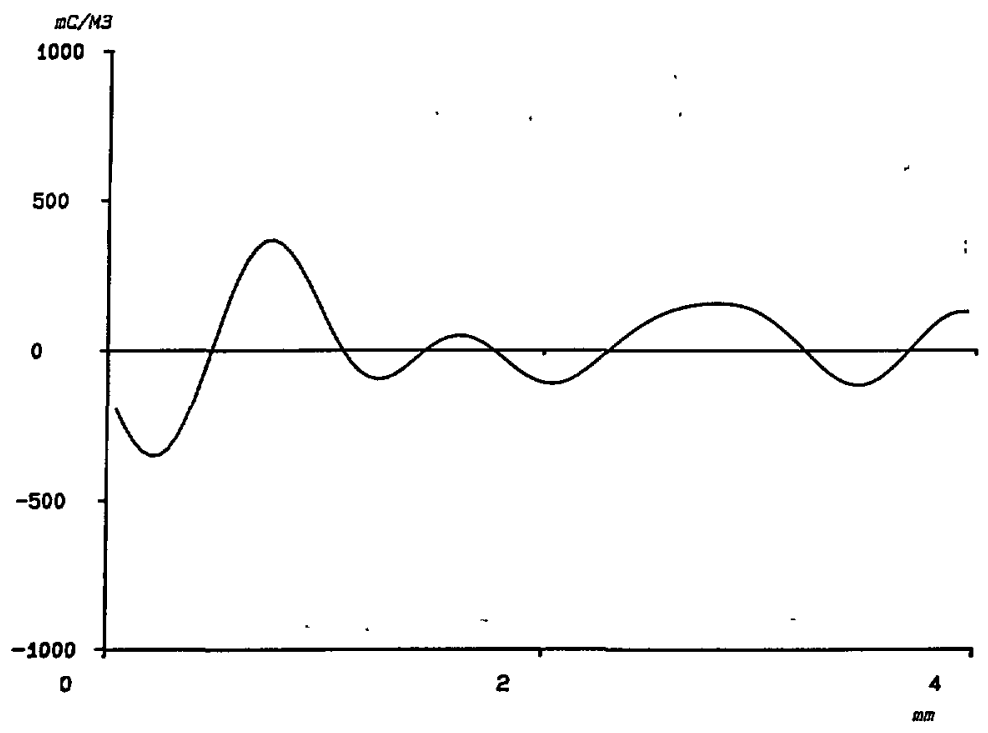

Fig. 3. - Distribution de charge d'espace dans un échantillon de PP (polypropylène) épais de 4,0 $\mathrm{mm}$ conservé 3 jours en court-circuit ; conditions de formation : voltage appliqué $40 \mathrm{kV}$ pendant 3 jours à $70^{\circ} \mathrm{C}$. Cathode à $x=0$ et anode à $x=4,0 \mathrm{~mm}$. Stockage à l'ambiante.

[Distribution of space charge density obtained in a $4.0 \mathrm{~mm}$ thick PP sample for a short circuiting storage time $t_{\mathrm{cc}}=2$ hours. Forming conditions : Applied voltage $40 \mathrm{kV}$ during 3 days at $70{ }^{\circ} \mathrm{C}$. Cathode at $x=0$, Anode at $x=4.0 \mathrm{~mm}$. Storage at room temperature.]

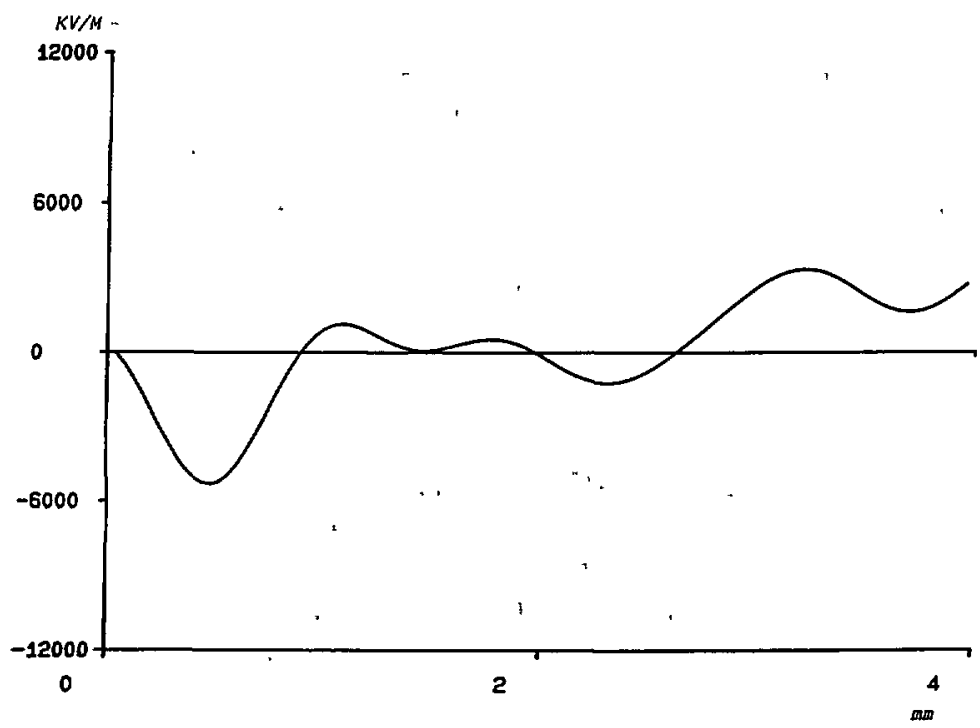

Fig., 4. - Distribution du champ électrique dans un échantillon de PP épais de 4,0 $\mathrm{mm}$ conservé 3 jours en court-circuit ; conditions de formation : voltage appliqué $40 \mathrm{kV}$ pendant 3 jours à $70{ }^{\circ} \mathrm{C}$. Cathode à $x=0$ et anode à $x=4,0 \mathrm{~mm}$. Stockage à l'ambiante.

[Distribution of electric field obtained in a $4.0 \mathrm{~mm}$ thick PP sample for a short circuiting storage time $t_{\mathrm{cc}}=2$ hours. Forming conditions: Applied voltage $40 \mathrm{kV}$. during 3 days at $70{ }^{\circ} \mathrm{C}$. Cathode at $x=0$, Anode at $x=4.0, \mathrm{~mm}$. Storage at room temperature.] 
Sur ces 2 exemples, on note la présence de zones successives de signes contraires; des alternances de régions positives et négatives ont été trouvées récemment dans des électrets de cire par un autre auteur [16] qui a utilisé une méthode tout à fait différente.

La figure 5 donne un courant expérimental mesuré et le courant simulé en partant des résultats trouvés pour la densité de charge. La concordance entre les deux courbes confirme la validité de notre méthode de déconvolution et la précision des résultats.

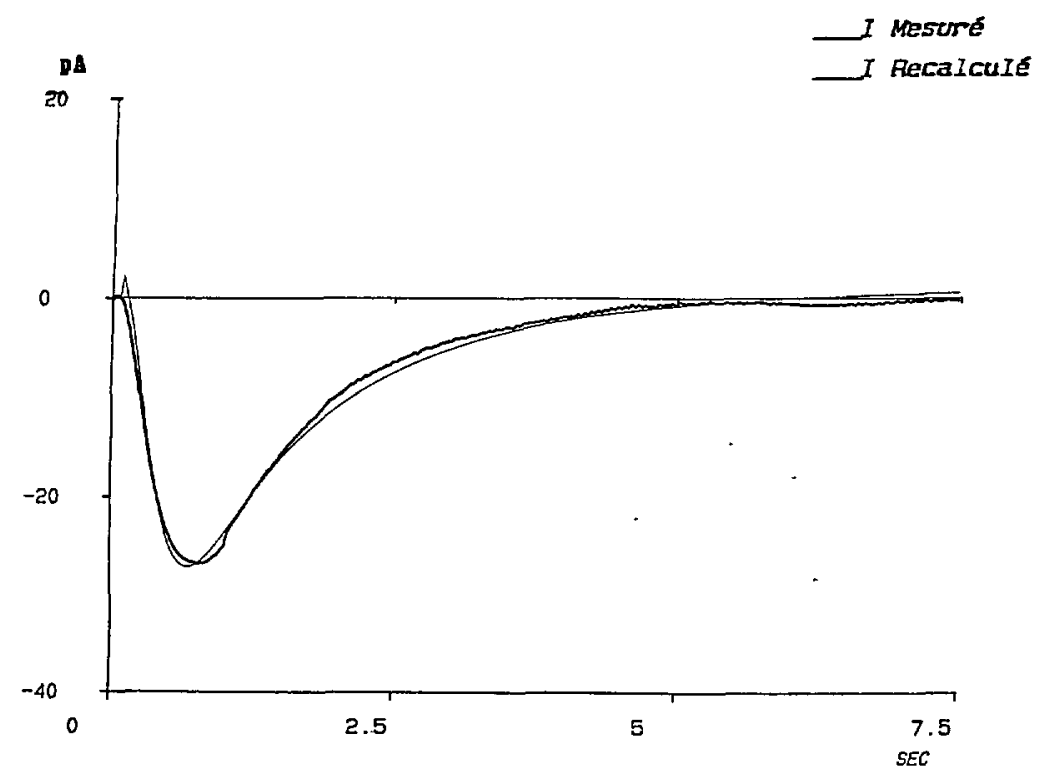

Fig. 5. - Comparaison entre le courant mesuré et le courant calculé à partir de la distribution trouvée dans un échantillon de PRC de $1,50 \mathrm{~mm}$ d'épaisseur. Conditions de formation: tension de $30 \mathrm{kV}$ pendant 2 jours à $70^{\circ} \mathrm{C}$, conservation en court-circuit : 8 jours à l'ambiante.

[Comparison between the measured current and the theoric one calculated from the space charge distribution found in a $1.50 \mathrm{~mm}$ thick PRC sample. Forming conditions : $30 \mathrm{kV}$ during 2 days at $70{ }^{\circ} \mathrm{C}$; the short circuiting storage time is 8 days at room temperature.]

\section{Conclusion.}

Nous venons de décrire la méthode de «l'onde thermique » que nous avons mise au point et perfectionnée ces deux dernières années. C'est une méthode simple dans son concept et peu coûteuse dans sa réalisation. Une partie importante du travail a résidé dans le développement de relations mathématiques exploitables entre le courant mesuré et les densités de charge d'espace. L'autre partie importante du travail a consisté à développer des logiciels adaptés au traitement numérique de déconvolution d'une équation intégrale faisant intervenir l'équation de la chaleur. Ces logiciels permettent d'obtenir la densité de charge $\rho(x)$ dans un isolant à partir du courant $I(t)$ que produit, dans le circuit extérieur, la diffusion d'un front thermique dont les caractéristiques sont déterminées par une mesure d'étalonnage. Par ailleurs, une fois la distribution de charge $\rho(x)$ calculée, ces mêmes logiciels permettent une vérification de la validité des résultats en recalculant le courant (simulé) que produirait la diffusion du front thermique considéré, sur cette distribution de charges. Il doit y avoir coïncidence entre le courant simulé et le courant mesuré au moins à la précision de la mesure près. 
Cette méthode est particulièrement bien appropriée aux études d'évolution de la charge d'espace liées aux problèmes de vieillissement des isolants, puisqu'elle est non destructive.

Son application à des structures en vraie grandeur comme les câbles a déjà été entreprise [17].

\section{Bibliographie}

[1] Croitoru Z., Space Charges in Dielectrics, Progr. Dielectr. 6 (1965) 105.

[2] Toureille A., $2^{\mathrm{e}}$ Conf. int. JICABLE Versailles, Sept. 1987, p. 98.

[3] Toureille A., Reboul J. P., Communication, "6th Symp. on Electrets " (ISE 6, Oxford) Sept. 1988.

[4] Toureille A., Reboul J. P., Simon C., Communication, $32^{\circ}$ Conf. Int. Grands Réseaux Electr. (C.I.G.R.E., Groupe 21, H. V. Insulated Cables, Paris) Sept. 1988.

[5] van Turnhout J., Thermally Stimulated Discharge of Polyethylene Electrets (Elsevier, Amsterdam) 1975.

[6] Reboul J. P., Toureille A., J. Polym. Sci., Polym. Phys. Ed. 22 (1984) 21.

[7] Collins R. E., Appl. Phys. Lett. 26 (1975) 675.

[8] von Seggern H., Appl. Phys. Lett. 33 (1978) 134.

[9] Laurenceau P., Ball J., Dreyfus G., Lewiner J., C. R. Acad. Sci. Paris B 283 (1976) 135.

[10] Rosno A. G., Gromov V. V., Pis ma V. Zh. T. Ph. (USSR) 5 (1979) 648.

[11] Alquie C., Dreyfus G., Lewiner J., Phys. Rev. Lett. 47 (1981) 1483.

[12] Sessler G. M., West J. E., Gerhard R., Polym. Bull. 6 (1981) 109.

[13] de Reggi A. S., Guttman C. M., Mopsik F. I., Davis G. T., Broadhurst M. G., Phys. Rev. Lett. 40 (1978) 413.

[14] Lang S. B., Das-Gupta D. K., Ferroelectrics 60 (1984) 23.

[15] Toureille A., Reboul J. P., Communication, Journées d'Etudes de la SEE (Isolants Electriques, Gif-sur-Yvette) 6 et 7 mars 1985.

[16] KACPRZYK R., J. Electrost. 22 (1989) 95.

[17] Toureille A., Sabir A., Reboul J. P., Berdala J. et Merle P., Rev. Phys. Appl. 25 (1990) 405. 